\title{
Klinische Relevanz von Tiermodellen für psychiatrische Störungen
}

Frauke Ohl und Saskia S. Arndt

10.1 Psychopathologische Verhaltenscharakteristika - 112

10.2 Angstverhalten $\mathbf{- 1 1 2}$

10.3 Biologische und klinische Relevanz von Tierverhalten - 113

Literatur - 113 
Psychiatrische Störungen sind komplexe pathologische Phänomene, die zu einer drastischen Beeinträchtigung des alltäglichen Lebens der betroffenen Patienten führen. Aufgrund ihrer Komplexität ist nach wie vor wenig über ihre Entstehung und die zugrunde liegenden Mechanismen bekannt. Daher sind Tiermodelle für psychiatrische Störungen auch heute noch unverzichtbar, und jedes Tiermodell, das unser Verständnis dieser Erkrankungen auch nur im Bezug auf Teilaspekte dieser Störungen erweitern kann, ist äußerst bedeutsam.

\subsection{Psychopathologische Verhaltenscharakteristika}

Die Diagnose psychiatrischer Störungen stützt sich maßgeblich auf die Feststellung bestimmter Verhaltenscharakteristika der Betroffenen. Einige dieser Symptome sind menschenspezifisch und daher nicht im Tiermodell darstellbar. Hierzu gehören v. a. solche Symptome, die ausschließlich subjektiv verbal darzulegen sind, wie z. B. die »Angst zu sterben « bei Patienten mit Panikstörungen oder das Wiedererleben traumatischer Ereignisse bei Patienten mit posttraumatischem Stresssyndrom. Trotzdem lassen sich auch bei Tieren psychopathologische Zustände feststellen, die dann als Verhaltensstörungen bezeichnet werden, und die zumindest als analog, wenn nicht homolog, zu bestimmten Symptomen psychiatrischer Störungen des Menschen einzustufen sind. In diesem Kontext ist jedoch die Erkenntnis von grundlegender Wichtigkeit, dass der unzweifelhafte Nutzen von Tiermodellen in der Möglichkeit zur Untersuchung distinkter Symptome psychiatrischer Störungen liegt und gleichzeitig auf diese begrenzt ist (Geyer u. Markou 1995): Die Komplexität einer psychiatrischen Störung im Tier vollständig zu modellieren, ist jedoch nicht möglich.

Wie bereits erwähnt, sind bei Tieren Verhaltensstörungen zu erkennen, die mit pathologischen Veränderungen des Verhaltens bei psychisch kranken Menschen vergleichbar sind. Außerdem ist die Effizienz bestimmter psychoaktiver Substanzen aus der Humanmedizin auch für Tiere belegt. Ebenso sind bestimmte Aspekte des normalen Verhaltens bei Mensch und Tier ähnlich, wobei dieses Verhalten zudem identische Steuerungsmechanismen aufweist (Overall 2000). Entsprechend sind Tiermodelle in der präklinischen Forschung zuverlässig einzusetzen bei

- der Untersuchung der zugrunde liegenden Mechanismen pathologisch veränderten Verhaltens,

- der Analyse der Verhaltenseffizienz potenzieller Behandlungsstrategien.

Dies wird im Folgenden am Beispiel des Angstverhaltens näher erläutert.

\subsection{Angstverhalten}

Evolutionär betrachtet ist Angst eine sehr ursprüngliche, essenzielle Emotion. Ihr adaptiver Charakter ermöglicht es, Unbe- kanntes vorsichtig zu erkunden, Reize qualitativ zu beurteilen und sich basierend auf Erlerntem durch Vermeidungsverhalten vor Gefahr zu schützen. Angst ist also nicht grundsätzlich als pathologisch zu verstehen, sondern als Emotion, die ein biologisch relevantes, dem Kontext entsprechendes Verhalten auslöst. Probleme entstehen erst dann, wenn Angst die biologische Relevanz verliert und nichtsituationsadäquates Verhalten hervorruft (Ohl et al. 2008). Dies ist bei Angststörungen der Fall (Rosen u. Schulkin 1998).

Pathologische Angst stellt somit nicht eine an sich krankhafte Emotion dar, sondern vielmehr eine normale Emotion, die nicht situationsgerecht ausgelöst wird und ihren adaptiven Charakter verloren hat. Es ist daher davon auszugehen, dass am Tiermodell gewonnene Erkenntnisse über die zentralnervöse Steuerung der »normalen « Angst für die Entstehung von Angststörungen bedeutsam sind. Die Stärke des Empfindens von Angst und somit auch das daraus resultierende Verhalten hängen letztlich immer von der individuellen und damit subjektiven Interpretation von Umwelteindrücken ab: Ein Mensch mit einer entsprechenden Angststörung empfindet beispielsweise eine normale Situation wie das Betreten eines offenen Platzes als lebensbedrohend. Während der Patient diese Empfindung verbal mitteilen kann, sind wir bei Tieren auf die Interpretation von Verhalten angewiesen.

\section{Ausdruck pathologischer Angst bei einem Tier}

Auch hier liegt der Schlüssel in der Kontextbezogenheit, d. h. der biologischen Relevanz des Verhaltens. So zeigen Nagetiere beispielsweise eine Tendenz, die offenen, hell erleuchteten Flächen eines ihnen unbekannten Areals zu vermeiden und zunächst dessen geschützte Bereiche zu erkunden. In einer Experimentalbox würde eine Maus dementsprechend zunächst einen Weg entlang der Wand wählen, bevor sie den zentralen, ungeschützten Bereich betritt (• Abb. 10.1a, b). Dieses Vermeidungsverhalten lässt sich auch bei frei lebenden Nagetieren beobachten; es stellt eine biologisch sinnvolle Explorationsstrategie dar, um dem Zugriff durch Beutegreifer zu entgehen. Um Futter oder einen Sexualpartner zu finden, ist es eine biologische Notwendigkeit, dass eine Maus auch offene Flächen durchqueren muss. Pathologische Angst unterscheidet sich also u. a. von normaler Angst durch ihren nichtadaptiven Charakter. Betritt die Maus nicht doch irgendwann die offene Fläche, so ist ihr Überleben bzw. der Fortbestand der Art nicht gesichert, und das Vermeidungsverhalten ist nicht länger biologisch sinnvoll.

Ein Tiermodell für pathologische Angst sollte sich also durch biologisch nichtadaptive Reaktionen auszeichnen. Ein pathologisch ängstliches Tier sollte sich also nicht an einen aversiven Reiz gewöhnen. Dieses Gewöhnungs- oder Anpassungsvermögen lässt sich z. B. testen, indem man ein Tier mehrfach ein und demselben aversiven Reiz aussetzt. In einer Experimentalbox würde eine pathologisch ängstliche Maus auch nach mehreren Tests noch stets den Weg entlang der Wand wählen und das offene Areal vermeiden. Durch die Verstärkung des aversiven Charakters der offenen Fläche, z. B. durch Einsatz einer hellen Lichtquelle, kann selbst eine Zunahme von angstbezogenem Vermeidungsverhalten auftreten (Sensitisierung; • Abb. 10.1c). 
a

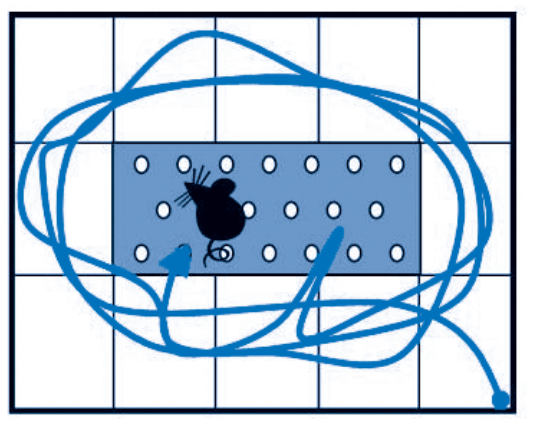

b

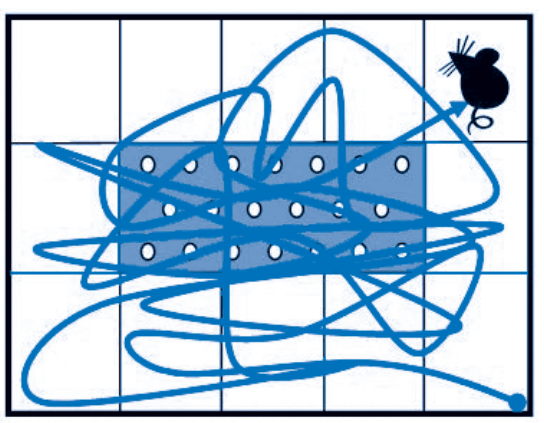

Vermeidungsverhalten

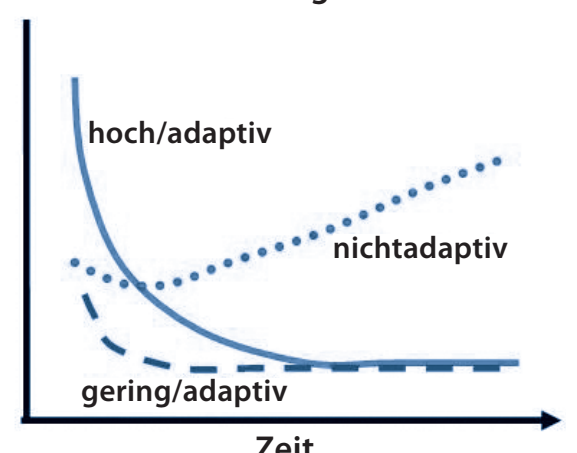

- Abb. 10.1a-c Nagetiere zeigen ein natürliches Vermeidungsverhalten gegenüber ungeschützten Arealen. Eine ängstliche Maus zeigt ein stärker ausgeprägtes Vermeidungsverhalten (a) als eine weniger ängstliche Maus (b), die in einem definierten Zeitraum mehr Zeit im ungeschützten Areal verbringt. (c) Nagetiere, die bei der wiederholten Konfrontation mit einem

Analog hierzu ist auch das Verhalten eines an einer Angststörung leidenden Menschen in bestimmten Situationen unangemessen und nichtadaptiv und wirkt sich daher nachteilig auf seine Lebensqualität aus.

\subsection{Biologische und klinische Relevanz von Tierverhalten}

Die biologische Relevanz des Verhaltens eines Tieres steht also in unmittelbarem Zusammenhang mit seiner klinischen Relevanz als Tiermodell. Hieraus folgt zwingend, dass die zur Verhaltensanalyse bei Labortieren eingesetzten Tests die Untersuchung biologisch relevanten Verhaltens erlauben müssen. Diese Grundvoraussetzung ist jedoch in vielen artifiziellen Testsituationen nicht gegeben. Um die biologische Relevanz des Verhaltens von Versuchstieren einschätzen zu können, sind valide Testsysteme (van der Staay et al. 2009) erforderlich, die es dem Tier erlauben, ein möglichst breites Verhaltensspektrum zu zeigen und die - hierauf basierend - detaillierte ethologische Verhaltensanalysen ermöglichen. Dies ist nur auf der Basis von Testverfahren möglich, die eine Vielzahl von Verhaltensdimensionen erfassen, z. B. Angst, lokomotorische Aktivität, exploratives Verhalten und soziale Affinität (Lister 1990; Ohl et al. 2001; Rodgers et al. 1997). Solch eine differenzierte Charakterisierung des basalen Verhaltens von potenziellen Tiermodellen ist v. a. insofern von großer Bedeutung, als alle in einem Individuum repräsentierten Verhaltensdimensionen in enger Interaktion miteinander stehen ( $>$ Box: Angst oder Aktivität? Die Notwendigkeit der detaillierten Verhaltensanalyse).

Zudem erfordert die Untersuchung pathologischen Verhaltens eine Analyse des adaptiven Charakters bestimmter Verhaltensweisen. Ohne Erkenntnisse über die Dynamik - mit anderen Worten die zeitliche Veränderung - biologisch sinnvollen Verhaltens, sind Rückschlüsse im Bezug auf pathologische $\mathrm{Zu}$ stände nicht möglich. Die Analyse von Verhaltensdimensionen auf der Basis komplexer ethologischer Untersuchungen ist daher eine wichtige Grundlage für die Einschätzung der biolo- bestimmten aversiven Reiz keine (ausreichende) Verhaltensadaptation zeigen, spiegeln Charakteristika humaner pathologischer Angst wieder. Dieses Vermeidungsverhalten kann experimentell, z. B. durch den Einsatz einer zusätzlichen Lichtquelle, noch verstärkt werden (Sensitisierung)

gischen Relevanz des Verhaltens eines Tieres und somit für die Beurteilung der klinischen Relevanz eines Tiermodells.

Angst oder Aktivität?

Die Notwendigkeit der detaillierten Verhaltensanalyse

Ein Individuum, ob Mensch oder Tier, dessen allgemeine Aktivität deutlich reduziert ist, kann in seinem Angstverhalten nur schlecht eingeschätzt werden. Andererseits kann ein Individuum, das exploratorisch überdurchschnittlich aktiv - also neugierig - ist, möglicherweise seine Angst schneller überwinden und erscheint dadurch weniger ängstlich als ein »normal« neugieriger Artgenosse (Weiss et al. 1998).

\section{Literatur}

Geyer MA, Markou A (1995) Animal models of psychiatric disorders. In: Bloom FE, Kupfer DJ (eds) Psychopharmacology: The Fourth Generation of Progress. Raven, New York, pp 787-798

Lister RG (1990) Ethologically based animal models of anxiety disorders. Pharmacol Ther 46: 321-340

Ohl F, Sillaber I, Binder E et al (2001) Differential analysis of basal behavior and diazepam-induced alterations in C57BL/6 and BALB/c mice using the modified hole board. J Psychiatr Res 35: 147-154

Ohl F, Arndt SS, van der Staay FJ (2008) Pathological anxiety in animals. Vet J 175(1): 18-26

Overall KL (2000) Natural animal models of human psychiatric conditions: assessment of mechanism and validity. Prog Neuropsychopharmacol Biol Psychiatry 24: 727-776

Rodgers RJ, Cao BJ, Dalvi A, Holmes A (1997) Animal models of anxiety: an ethological perspective. Braz J Med Biol Res 30: 289-304

Rosen JB, Schulkin J (1998) From normal fear to pathological anxiety. Psychol Rev 105: 325-350

Van der Staay FJ, Arndt SS, Nordquist RE (2009) Evaluation of animal models of neurobehavioral disorders. Behav Brain Funct 5: 11

Weiss SM, Wadsworth G, Fletcher A, Dourish CT (1998) Utility of ethological analysis to overcome locomotor confounds in elevated maze models of anxiety. Neurosci Biobehav Rev 23: 265-271 
